of reprogramming proteins. Cell Stem Cell 4: 472-476.

Park $1 \mathrm{H}$, Zhao R, West JA, Yabuuchi A, Huo H, Ince TA et al (2008). Reprogramming of human somatic cells to pluripotency with defined factors. Nature 451: 141-146.

Takahashi K, Tanabe K, Ohnuki M, Narita M, Ichisaka T, Tomoda K et al (2007). Induction of pluripotent stem cells from adult human fibroblasts by defined factors. Cell 131: 861-872.

Takahashi K, Yamanaka S (2006). Induction of pluripotent stem cells from mouse embryonic and adult fibroblast cultures by defined factors. Cell 126: 663-676.

Yamanaka $S$ (2009). A fresh look at iPS cells. Cell 137: 13-17.

Yu J, Vodyanik MA, Smuga-Otto K, AntosiewiczBourget J, Frane JL, Tian S et al (2007). Induced pluripotent stem cell lines derived from human somatic cells. Science 318: 1917-1920.

Neuropsychopharmacology Reviews (2010) 35 346-348; doi: I0.1038/npp.2009. 108

\section{Advances in PET analyses of stress and dopamine}

A deregulation of the stress response is a potential etiological factor in the development and relapse of dopamine (DA)-related human disorders, including addiction, drug-induced psychosis, and schizophrenia. Schizophrenia is characterized by a chronic relapsing nature and vulnerability to exacerba- tion following stress or dopaminergic drugs. Indeed, the most replicated finding so far in schizophrenia positron emission tomography (PET) research has been an increased DA release after the administration of amphetamine, and this DA increase has been associated with positive psychotic symptoms and active stages of the illness. As increased stimulantinduced DA release is a hallmark of sensitization, several pieces of evidence support the view that schizophrenia is associated with a process of dopamine sensitization (Laruelle and Abi-Dargham, 1999).

Psychosis sufferers themselves often cite a link between the experience of stress and psychotic symptoms. The stress-vulnerability model suggests that an endogenous, organic diathesis or vulnerability interacts with internal or external stressors in the development of psychotic disorders. However, the underlying neurobiological condition/event that results in an exaggerated response to stressors is unknown. One proposed mechanism is neurochemical sensitization of the DA system-dopamine sensitization-, whereby repeated exposure to sensitizing life stressors (or DA drugs) progresses into increased stress-associated DA release. Hence, during active periods of the illness (that is, prodromal phase, initial episode, and subsequent relapses), dopaminergic neurons may be hyperresponsive to environmental stimuli, and exposure to even moderate levels of stress (or DA drugs) produces excessive DA release, precipitating illness in vulnerable individuals and relapse in those with schizophrenia.

The phenomenon of DA sensitization to psychostimulants is subjected to cross-sensitization with stress. It is well established that repeated stress (maternal separation, social-defeat stress, foot shock, tail pinch, restraint) induces heightened sensitivity to low doses of dopaminergic drugs; and conversely, repeated dopaminergic drug exposure increases the stress response in animals. Consistent with these findings, prenatal and postnatal stress is associated with increased drug-induced DA release, and different types of acute and repeated stress increase DA release (Abercrombie et al, 1989; Kalivas et al, 1986). Similarly, recent studies in humans using PET have shown increased DA release after either a psychosocial or a metabolic stress task (Adler et al, 2000; Pruessner et al, 2004). However, this finding was not replicated in another study, which used a different stress paradigm, with no
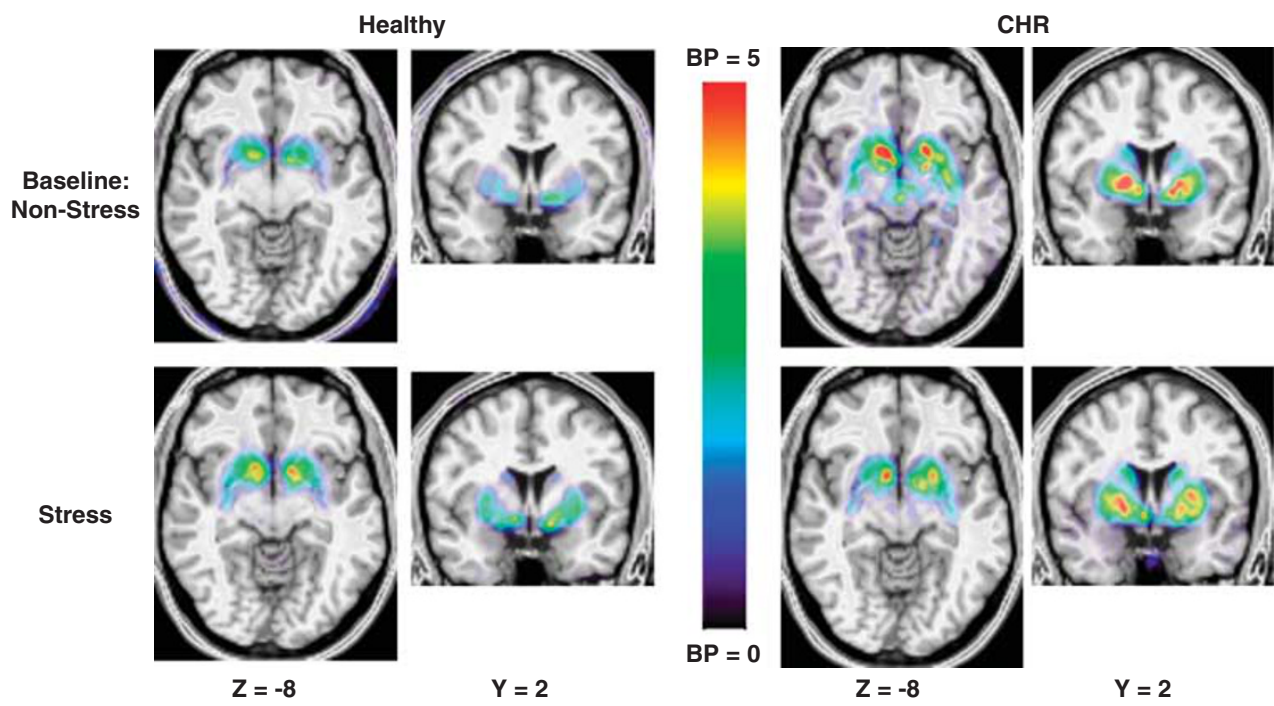

Figure 1. Representative figure showing decreased $\left[{ }^{11} \mathrm{C}\right]-(+)-\mathrm{PHNO} B \mathrm{P}_{\mathrm{ND}}$ in $\mathrm{CHR}$ following stress suggesting increased DA displacement, as compared with HV. 
apparent negative feedback (that is, social stress) (Montgomery et al, 2006).

The next logical step is to test whether stress produces a deregulated DA response in clinical populations, such as those with psychotic disorders, addictions, as well as in those at risk. We tested both drug-free patients with schizophrenia and clinical high risk (CHR) for psychosis (that is, putatively prodromal) diagnosed with the Criteria of Prodromal Syndromes in a two-scan protocol: one while doing a Sensory Motor Control Task and another one while doing the Montreal Imaging Stress Task. Our pilot data suggests an increased stressinduced DA release as compared with matched healthy volunteers (HVs) (Figure 1). This kind of studies has important theoretical and clinical implications regarding the prevention of relapse and efforts to abort or delay conversion to psychosis.

\section{Romina Mizrahi ${ }^{1,2}$}

${ }^{1} \mathrm{CAMH}, \mathrm{PET}$ centre, Toronto, ON, Canada and

${ }^{2}$ Department of Psychiatry, Faculty of Medicine, University of Toronto, Toronto, ON, Canada

E-mail: romina.mizrahi@camhpet.ca

\section{DISCLOSURE}

The author declares no conflict of interest.

Abercrombie ED, Keefe KA, DiFrischia DS Zigmond MJ (1989). Differential effect of stress on in vivo dopamine release in striatum, nucleus accumbens, and medial frontal cortex. J Neurochem 52: 1655-1658.

Adler CM, Elman I, Weisenfeld N, Kestler L, Pickar D, Breier A (2000). Effects of acute metabolic stress on striatal dopamine release in healthy volunteers. Neuropsychopharmacology 22: 545-550.

Kalivas PW, Richardson-Carlson R, Van Orden G (1986). Cross-sensitization between foot shock stress and enkephalin-induced motor activity. Biol Psychiatry 21: 939-950.

Laruelle M, Abi-Dargham A (1999). Dopamine as the wind of the psychotic fire: new evidence from brain imaging studies. J Psychopharmacol 13: 358-371.

Montgomery AJ, Mehta MA, Grasby PM (2006). Is psychological stress in man associated with increased striatal dopamine levels? A [11C]raclopride PET study. Synapse 60: 124-131.

Pruessner JC, Champagne F, Meaney MJ, Dagher A (2004). Dopamine release in response to a psychological stress in humans and its relationship to early life maternal care: a positron emission tomography study using [11C]raclopride. J Neurosci 24: 2825-2831.

Neuropsychopharmacology Reviews (2010) 35, 348-349; doi: I0.1038/npp.2009.132

\section{Imaging and genetics advances in understanding geriatric depression}

Over a decade has passed since publication of the vascular depression hypothesis that cerebrovascular disease may predispose, precipitate, or perpetuate some geriatric depressive syndromes (Alexopoulos et al, 1997). This construct has catalyzed much research, largely focused on structural neuroimaging, clinical neuropsychology, and in last few years, genetics and cognitive neuroscience. Recent investigations have combined advanced neuroimaging techniques and genetic analyses to delineate key structural and functional findings in late-life depression (LLD).

Vascular brain changes on imaging studies have long been associated with LLD. Genetic analysis of vascular changes is an emerging field, and recent work in older populations has focused on polymorphisms associated with (1) psychiatric disease in younger adults or (2) vascular risk factors. We have previously reported links between BDNF (brain-derived neurotrophic factor) polymorphisms (Taylor et al, 2008) and serotonin transporter-linked polymorphic region (5HTTLPR) polymorphisms (Steffens et al, 2008) and white matter hyperintensity (WMH) volumes, supporting theories that these genes also have a role in LLD. The notion that WMHs are vascular in nature led our group to explore relationships between WMH volume and genes associated with systemic vascular risk. These include renin-angiotensin system genes, such as the angiotensin II receptor, vascular types 1 and 2 (AGTR1 and AGTR2) genes. In addition to reports linking AGTR1 polymorphisms to LLD outcomes, we have also found genderspecific associations between AGTR polymorphisms and WMH progression (Taylor et al, 2009). Similarly, the methylenetetrahydrofolate reductase (MTHFR) gene, another vascular risk gene associated with folate and homo- cysteine metabolism, may be associated with LLD, and we are examining MTHFR and other genes related to folate metabolism in the occurrence of white matter lesions in LLD. Our studies continue to examine the role of vascular risk genes in LLD, focusing on their influence on brain structure and cognition.

Consistent with the vascular depression hypothesis is the notion the LLD is associated with disruption in frontalstriatal circuitry. These abnormalities in the prefrontal cortex, striatum, and frontal-striatal white matter tracks are also likely related to genetic differences. A previous study noted a link between the 5HTTLPR short allele and caudate volume in LLD. We recently reported an interaction between two genes, COMT (catechol-O-methyltransferase) and MTHFR, and putamen volume reduction in LLD (Pan et al, 2009). Identification of genes associated with prefrontal cortical structure and function remains an active area of inquiry.

Beyond the vascular depression hypothesis, another avenue of genetic/neuroimaging LLD research examines the observed link between depression and later dementia. The hippocampus is a key structure related to both LLD pathophysiology and increased dementia risk. The APOE gene is associated with both depression and Alzheimer's disease, and APOE genotype has been associated with specific shape differences in geriatric depression (Qiu et al, 2009). Allelic differences in the 5HTTLPR gene have also been associated with hippocampal volume in LLD by several groups.

Advances in both neuroimaging technologies and genetic assessment allow us to investigate the pathophysiology of affective disorders. Existing paradigms of vascular depression and depression as dementia risk make LLD ideally suited for future genetic/imaging studies.

\section{David C Steffens ${ }^{1}$}

${ }^{1}$ Department of Psychiatry and Behavioral Sciences, Duke University Medical Center, Durham, NC, USA E-mail: steff001@mc.duke.edu 